\title{
NONRESONANCE CONDITIONS FOR FOURTH ORDER NONLINEAR BOUNDARY VALUE PROBLEMS
}

\section{DE COSTER, C. FABRY, F. MUNYAMARERE}

Département de Mathématique Université Catholique de Louvain Chemin du Cyclotron 2 B-1348 Louvain-la-Neuve Belgium

(Received February 6, 1992)

ABSTR $\Lambda$ CT. This paper is devoted to the study of the problem

$$
\begin{gathered}
u^{(4)}=f\left(t, u, u^{\prime}, u^{\prime \prime}, u^{\prime \prime \prime}\right), \\
u(0)=u(2 \pi), u^{\prime}(0)=u^{\prime}(2 \pi), u^{\prime \prime}(0)=u^{\prime \prime}(2 \pi), u^{\prime \prime \prime}(0)=u^{\prime \prime \prime}(2 \pi) .
\end{gathered}
$$

We assume that $f$ can be written under the form

$$
\begin{aligned}
f\left(t, u, u^{\prime}, u^{\prime \prime}, u^{\prime \prime \prime}\right)=f_{2}\left(t, u, u^{\prime}, u^{\prime \prime}, u^{\prime \prime \prime}\right) u^{\prime \prime}+f_{1}\left(t, u, u^{\prime}, u^{\prime \prime}, u^{\prime \prime \prime}\right) u^{\prime} \\
\\
+f_{0}\left(t, u, u^{\prime}, u^{\prime \prime}, u^{\prime \prime \prime}\right) u+r\left(t, u, u^{\prime}, u^{\prime \prime}, u^{\prime \prime \prime}\right)
\end{aligned}
$$

where $r$ is a bounded function. We obtain existence conditions related to uniqueness conditions for the solution of the linear problem

$$
\begin{gathered}
u^{(4)}=a u+b u^{\prime \prime} \\
u(0)=u(2 \pi), u^{\prime}(0)=u^{\prime}(2 \pi), u^{\prime \prime}(0)=u^{\prime \prime}(2 \pi), u^{\prime \prime \prime}(0)=u^{\prime \prime \prime}(2 \pi) .
\end{gathered}
$$

KEY WORDS AND PHRASES. Nonresonance conditions, fourth order periodic BVP, eigenlines. AMS SUBJECT CLASSIFICATION CODE. 34C25, 34B99, 34L30.

\section{INTRODUCTION}

This paper is devoted mainly to the study of the fourth order periodic boundary value problem

$$
\begin{gathered}
u^{(4)}=f\left(t, u, u^{\prime}, u^{\prime \prime}, u^{\prime \prime \prime}\right), \\
u(0)=u(2 \pi), u^{\prime}(0)=u^{\prime}(2 \pi), u^{\prime \prime}(0)=u^{\prime \prime}(2 \pi), u^{\prime \prime \prime}(0)=u^{\prime \prime \prime}(2 \pi) .
\end{gathered}
$$

Boundary value problems for fourth order ordinary differential equations have received considerable interest recently. As a starting point, we state a result of Y. Yang [22] about a nonlinear problem with Dirichlet-type boundary conditions :

$$
\begin{gathered}
u^{(4)}=f\left(t, u, u^{\prime \prime}\right), \\
u(0)=u(\pi)=0, u^{\prime \prime}(0)=u^{\prime \prime}(\pi)=0 .
\end{gathered}
$$

Y. Yang proves the existence of a solution under the assumption that

$$
|f(t, u, v)| \leq a|u|+b|v|+c
$$

with

$$
a+b<1 .
$$


With the same boundary conditions, Y. Yang [23] has also established the existence of a solution for the equation

$$
u^{(\cdot)}=g\left(t, u, u^{\prime}, u^{\prime \prime}, u^{\prime \prime \prime}\right) u+h\left(t, u, u^{\prime}, u^{\prime \prime}, u^{\prime \prime \prime}\right)
$$

assuming that, for $U \in \mathbb{R}^{4}, h(t, U)$ is bounded and there exists $k \in \mathbb{N}$ such that

$$
k^{4} \lesssim \inf _{U} g(t, U) \leq \sup _{U} g(t, U) \lesssim(k+1)^{4} \quad \text { a.e. on }[0, \pi],
$$

where the notation $\lesssim$ means that the inequality is strict on a subset of positive measure. This last condition can be clearly read as a non-interference condition of the nonlinearity with respect to the spectrum of the operator $u \mapsto u^{(4)}$, subject to the boundary conditions (1.4). Recently, M. Del Pino and R. Manasevich [5] have extended Y. Yang's [22] result. They prove the existence of a solution for (1.3), (1.4), assuming that

$$
|f(t, u, v)-(\alpha u+\beta v)| \leq a|u|+b|v|+c
$$

the numbers $\alpha, \beta \in \mathbb{R}$ and $a, b, c \in \mathbb{R}_{+}$being such that

$$
\begin{gathered}
\frac{\alpha}{k^{4}}-\frac{\beta}{k^{2}} \neq 1 \text { for all } k \in \mathbb{N}^{*}, \\
a \max _{k \in \mathbb{N}^{*}} \frac{1}{\left|k^{4}-\alpha+\beta k^{2}\right|}+b \max _{k \in \mathbb{N}^{*}} \frac{k^{2}}{\left|k^{4}-\alpha+\beta k^{2}\right|}<1 .
\end{gathered}
$$

The aim of this paper is to provide analogous results for the periodic boundary value problem (1.1), (1.2). Those results will however be obtained by a method of proof different from that of the above-mentioned papers.

Before discussing the problem further, let us mention a few recent contributions to fourth order boundary value problems, namely by A.R. Aftabizadeh [1], R.P. Agarwal [2], C. Fabry and F. Munyamarere [6], C. Gupta [7-13], G. Metzen [17], L. Sanchez [18], J.D. Schuur [19].

In order to obtain sharp existence results for the problem (1.1), (1.2), it seems appropriate to relate it to the two-parameter eigenvalue problem

$$
\begin{gathered}
u^{(4)}=a u+b u^{\prime \prime} \\
u(0)=u(2 \pi), u^{\prime}(0)=u^{\prime}(2 \pi), u^{\prime \prime}(0)=u^{\prime \prime}(2 \pi), u^{\prime \prime \prime}(0)=u^{\prime \prime \prime}(2 \pi)
\end{gathered}
$$

about which it is an easy matter to prove the following result.

LEMMA 1. The problem (1.6), (1.7) has a nontrivial solution if and only if there exists $k \in \mathbb{N}$ such that

$$
k^{4}=a-b k^{2} .
$$

For $k \in \mathbb{N}$, we will call the set $L_{k}=\left\{(a, b) \in \mathbb{R}^{2} \mid k^{4}=a-b k^{2}\right\}$ an eigenline of (1.6), (1.7). The condition (1.5) then means that the point $(\alpha, \beta)$ does not lie on any eigenline.

We will treat problem (1.1), (1.2) by separating two cases, depending on the form of $f$. In the first case, discussed in section 3 , we will assume that $f$ can be written under the form

$$
f\left(t, u, u^{\prime}, u^{\prime \prime}, u^{\prime \prime \prime}\right)=\left(g\left(t, u, u^{\prime}\right) u^{\prime}\right)^{\prime}+h\left(t, u, u^{\prime}, u^{\prime \prime}, u^{\prime \prime \prime}\right) u+r\left(t, u, u^{\prime}, u^{\prime \prime}, u^{\prime \prime \prime}\right) .
$$

where $r\left(t, u, u^{\prime}, u^{\prime \prime}, u^{\prime \prime \prime}\right)$ is a bounded function. We will be able to prove the existence of a solution under the assumption that the point $(g(t, x, y), h(t, x, y, z, w))$ always lies in a rectangle with sides parallels to the axes and which does not intersect any eigenline. This kind of result can be considered as an extension of the results of Y. Yang [23] (with obvious modifications, since the boundary conditions are different). It is worthwhile to adapt that result in the case where the point $(g(t, x, y), h(t, x, y, z, w))$ is located at the left of all eigenlines. That situation is also dealt 
with in section 3; the results obtained there generalize results of C. Gupta and J. Mawhin [13]. The above results have a simple geometric description in terms of eigenlines; however, they have the drawback to rely on the decomposition (1.8) of $f$, for which we are unable to give practical hypotheses ensuring its existence. Therefore, in section 4, we work with a different decomposition of $f$, namely

$$
f\left(t, u, u^{\prime}, u^{\prime \prime}, u^{\prime \prime \prime}\right)=f_{2}\left(t, u, u^{\prime}, u^{\prime \prime}, u^{\prime \prime \prime}\right) u^{\prime \prime}+f_{0}\left(t, u, u^{\prime}, u^{\prime \prime}, u^{\prime \prime \prime}\right) u+r\left(t, u, u^{\prime}, u^{\prime \prime}, u^{\prime \prime \prime}\right) .
$$

where $r\left(t, u, u^{\prime}, u^{\prime \prime}, u^{\prime \prime \prime}\right)$ is a bounded function. Such a decomposition can be obtained, for instance, on the basis of an hypothesis of the type

$$
|f(t, u, v, u, z)-p u-q w| \leq \alpha(t)|u|+\beta(t)|w|+\gamma(t) .
$$

That kind of condition has been used in M. Del Pino and R. Manasevich [5], C. Fabry and F. Munyamarere [6], Y. Yang [22]. Unfortunately, the existence conditions obtained with the decomposition (1.9) are not as nice as those obtained with (1.8). In particular, we are unable to answer a question raised by M. Del Pino and R. Manasevich : can the existence of a solution be proven under the hypothesis that the point $\left(f_{0}, f_{2}\right)$ lies in a rectangle which does not intersect the eigenlines? In the sequel, we will refer to the case where $f$ admits the decomposition (1.8) as the "symmetric case", whereas the decomposition (1.9) will be referred to as the "nonsymmetric case".

Our method of proof is inspired by a method used by J. Mawhin and J.R. Ward [15] [16] for the periodic boundary value problem

$$
\begin{gathered}
-u^{\prime \prime}=f(t, u), \\
u(0)=u(2 \pi), u^{\prime}(0)=u^{\prime}(2 \pi) .
\end{gathered}
$$

They prove the existence of a solution, assuming that, for some $n \in \mathbb{N}$,

$$
n^{2} \lesssim a(t) \leq \liminf _{|u| \rightarrow \infty} \frac{f(t, u)}{u} \leq \limsup _{|u| \rightarrow \infty} \frac{f(t, u)}{u} \leq b(t) \lesssim(n+1)^{2} .
$$

Their proof relies on the use of a coercive quadratic form. More precisely, they use the fact that the Sobolev space $H_{2 \pi}^{1}$ admits a decomposition $H_{2 \pi}^{1}=\bar{H} \oplus \tilde{H}$ with $\operatorname{dim} \bar{H}<\infty$ such that, for all $\bar{u} \in \bar{H}, \tilde{u} \in \tilde{H}$, we have

$$
\int_{0}^{2 \pi}\left[\tilde{u}^{\prime 2}-b(t) \tilde{u}^{2}\right]-\int_{0}^{2 \pi}\left[\bar{u}^{\prime 2}-a(t) \bar{u}^{2}\right] \geq \delta\|\bar{u}+\tilde{u}\|_{H^{1}}^{2},
$$

for some $\delta>0$.

In section 2 , we introduce an abstract version of such type of hypothesis to obtain an existence result for the general nonlinear equation $L u=N u$; that result will then be applied to the periodic fourth order boundary value problem.

At the end of this introduction let us fix some notations. We will use the following spaces:

$$
\begin{gathered}
L^{k}(0,2 \pi)=\left\{u:[0,2 \pi] \rightarrow \mathbb{R} \text { measurable }\left.\left|\int_{0}^{\pi}\right| u(t)\right|^{k} d t<\infty\right\}, \\
W^{k, p}(0,2 \pi)=\{u:[0,2 \pi] \rightarrow \mathbb{R} \mid \text { for } j=0, \ldots, k-1, \\
\left.u^{(\jmath)} \text { is absolutely continuous and } \int_{0}^{\pi}\left|u^{(k)}(t)\right|^{p} d t<\infty\right\}, \\
H^{k}(0,2 \pi)=W^{k, 2}(0,2 \pi), \\
H_{2 \pi}^{k}=\left\{u \in H^{k}(0,2 \pi) \mid u(0)=u(2 \pi), \ldots, u^{(k-1)}(0)=u^{(k-1)}(2 \pi)\right\}, \\
\mathcal{C}^{k}[0,2 \pi]=\{u:[0,2 \pi] \rightarrow \mathbb{R} k-\text { times continuously differentiable }\} .
\end{gathered}
$$


We will often only write $\mathcal{C}^{k}, L^{k}, W^{k, p}, H^{k}$. The norms on those spaces are defined as usual; on $I^{2}$, we will use the norm $\|u\|_{H^{2}} \cdot\|u\|_{L^{2}}+\left\|u^{\prime \prime}\right\|_{L^{2}}$.

Finally we will say that a function $f:[0,2 \pi] \times \mathbb{R}^{n} \rightarrow \mathbb{R}$ satisfies $L^{p}$-Carathéodory conditions if

1) for each $u \in \mathbb{R}^{n}, f$ is measurable in $t$;

2) for almost every $t \in[0,2 \pi], f$ is continuous in $u$;

3) for every $R>0$, there exists a function $h_{R} \in L^{p}(0,2 \pi)$ such that $|f(t, u)| \leq h_{R}(t)$ a.e. $t \in[0,2 \pi]$ and for each $u \in \mathbb{R}^{n}$ with $\|u\|<R$.

\section{ABSTRACT RESULTS}

In this section, we first present an existence result for the abstract nonlinear equation

$$
L u=N u
$$

that result is a generalization of Theorem 3 in C. De Coster, C. Fabry and P. Habets [4].

Let $H$ be a Hilbert space, $X$ and $V$ be normed spaces such that $H$ is continuously embedded in $V^{\prime}$, the dual of $V$. We denote by $\langle.,$.$\rangle the pairing in V$. Let $A, B: X \rightarrow V$ be linear, symmetric operators such that for all $u \in X \cap V^{\prime}$

$$
\langle A u, u\rangle \leq\langle B u, u\rangle .
$$

Denote by $\mathcal{F}(X, V)$ the set of linear, symmetric operators $S: X \rightarrow V$ such that, for all $u \in X \cap V^{\prime}$

$$
\langle A u, u\rangle \leq\langle S u, u\rangle \leq\langle B u, u\rangle .
$$

The theorem below uses coincidence degree arguments; for a presentation of that theory and the definition of $L$-compacity and $L$-complete continuity, the reader is referred to J. Mawhin [14].

THEOREM 2. Let $L: \operatorname{dom} L \subset H \cap X \rightarrow V$ be a linear, symmetric, Fredholm operator of index zero and $N: X \rightarrow V$ be a $L$-completely continuous operator. Assume that :

(a) the operators $A, B$ are $L$-compact;

(b) the bilinear forms $\overline{\mathcal{A}}, \overline{\mathcal{B}}, \overline{\mathcal{L}}: \operatorname{dom} L \times \operatorname{dom} L \rightarrow \mathbb{R}$ respectively defined by $\overline{\mathcal{A}}(u, v)=\langle A u, v\rangle$, $\overline{\mathcal{B}}(u, v)=\langle B u, v\rangle, \overline{\mathcal{L}}(u, v)=\langle L u, v\rangle$ admit continuous extensions to $H \times H$ denoted by $\mathcal{A}, \mathcal{B}, \mathcal{L}$;

(c) for every $K>0$, there exists $M>0$ such that if $u$ is a solution of

$$
L u=\lambda N u+(1-\lambda) \frac{A+B}{2} u
$$

with $\|u\|_{H}<K$ and $\lambda \in[0,1]$, then $\|u\|_{X}<M$.

If, moreover,

(i) there exist $\mathcal{D}(.,$.$) a positive definite bilinear form and a decomposition H=\bar{H} \oplus \tilde{H}$ with dim $\bar{H}<\infty$ such that, for any $\bar{u} \in \bar{H}, \tilde{u} \in \tilde{H}$,

$$
[\mathcal{L}(\tilde{u}, \tilde{u})-\mathcal{B}(\tilde{u}, \tilde{u})]-[\mathcal{L}(\bar{u}, \bar{u})-\mathcal{A}(\bar{u}, \bar{u})] \geq \mathcal{D}(\bar{u}+\tilde{u}, \bar{u}+\tilde{u}) ;
$$

(ii) the operator $N$ admits the decomposition

$$
N u=G(u) u+Q(u)
$$

where for all $u \in X, G(u) \in \mathcal{F}(X, V)$, the bilinear form $\overline{\mathcal{G}}(x)$ defined on dom $L \times \operatorname{dom} L$ by $\overline{\mathcal{G}}(x)(u, v)=\langle G(x) u, v\rangle$ admits a continuous extension to $H \times H$ denoted by $\mathcal{G}(x)$ and there exists $R>0$ such that, for all $u \in \operatorname{dom} L, u=\bar{u}+\tilde{u}$ with $\bar{u} \in \bar{H}, \tilde{u} \in \tilde{H}$ and $\|u\|_{H} \geq R$,

$$
\langle Q(\bar{u}+\tilde{u}), \tilde{u}-\bar{u}\rangle<\mathcal{D}(\bar{u}+\tilde{u}, \bar{u}+\tilde{u}) .
$$

Then there exists at least one solution $u$ of

$$
L u=N u .
$$


PROOF. By the coincidence degree theory. we only have to find an a priori bound in $X$ for the solutions of

$$
L u=\lambda N u \mid(1-\lambda) \frac{A+B}{2} u, \quad \lambda \in[0,1] .
$$

By hypothesis (c), it is enough to prove that for all $u$ solution of (2.3), we have $\|u\|_{H}<R$.

Assume by contradiction that there is a solution $(\lambda, u)$ of $(2.3)$ with $\|u\|_{H} \geq R$. Let us write $u=\bar{u}+\tilde{u}$ with $\bar{u} \in \bar{H}$ and $\tilde{u} \in \tilde{H}$, let $N_{\lambda}:=\lambda N+(1-\lambda)(A+B) / 2, \mathcal{G}_{\lambda}(u)=\lambda \mathcal{G}(u)+(1-\lambda)(\mathcal{A}+\mathcal{B}) / 2$. Multiplying (2.3) by $\tilde{u}-\bar{u}$, we obtain using (2.1) and (2.2),

$$
\begin{aligned}
0 & =\left\langle L u-N_{\lambda} u, \tilde{u}-\bar{u}\right\rangle \\
& =\left[\mathcal{L}(\tilde{u}, \tilde{u})-\mathcal{G}_{\lambda}(u)(\tilde{u}, \tilde{u})\right]-\left[\mathcal{L}(\bar{u}, \bar{u})-\mathcal{G}_{\lambda}(u)(\bar{u}, \bar{u})\right]-\lambda\langle Q(u), \tilde{u}-\bar{u}\rangle \\
& \geq[\mathcal{L}(\tilde{u}, \tilde{u})-\mathcal{B}(\tilde{u}, \tilde{u})]-[\mathcal{L}(\bar{u}, \bar{u})-\mathcal{A}(\bar{u}, \bar{u})]-\lambda\langle Q(u), \tilde{u}-\bar{u}\rangle>0
\end{aligned}
$$

which gives a contradiction and proves that $\|u\|_{H}<R$.

The existence of a decomposition $H=\bar{H} \oplus \tilde{H}$ such that (2.1) holds can be obtained by means of the following proposition which is proved in C. De Coster, C. Fabry and P. Habets [4].

PROPOSITION 3. Let $H$ be a vector space and $\mathcal{L}, \mathcal{A}, \mathcal{B}$ be real, bilinear, symmetric forms on $H$. Assume that

(i) there is some $m \in \mathbb{R}$ such that

$$
\mathcal{C}=\mathcal{L}+m \mathcal{B}-(1+m) \mathcal{A}
$$

is a scalar product which makes $H$ a Hilbert space;

(ii) $\mathcal{B}-\mathcal{A}$ is positive definite, i.e.

$$
(\forall u \in H, u \neq 0),(\mathcal{B}-\mathcal{A})(u, u)>0
$$

(iii) for any sequence $\left(u_{k}\right)_{k}$ such that $u_{k} \stackrel{\mathcal{C}}{\rightarrow} u$, one has

$$
(\mathcal{B}-\mathcal{A})\left(u_{k}, u_{k}\right) \rightarrow(\mathcal{B}-\mathcal{A})(u, u)
$$

Then the following equivalences hold :

(a) the interval $[0,1]$ does not contain eigenvalues $\lambda$ of the problem

$$
\forall v \in H, \mathcal{L}(u, v)=\lambda \mathcal{B}(u, v)+(1-\lambda) \mathcal{A}(u, v)
$$

(b) there exists $\delta>0, \bar{H} \subset H$ and $\tilde{H} \subset H$ such that $\operatorname{dim} \bar{H}<\infty, H=\bar{H} \oplus \tilde{H}$ and, for any $\bar{u} \in \bar{H}, \tilde{u} \in \tilde{H}$, one has

$$
(\mathcal{L}-\mathcal{B})(\tilde{u}, \tilde{u})-(\mathcal{L}-\mathcal{A})(\bar{u}, \bar{u}) \geq \delta \mathcal{C}(\bar{u}+\tilde{u}, \bar{u}+\tilde{u})
$$

(c) for any real bilinear symmetric form $\mathcal{S}$ on $H$ such that $\mathcal{A} \leq \mathcal{S} \leq \mathcal{B}$, i.e. such that

$$
\forall u \in H, \mathcal{A}(u, u) \leq \mathcal{S}(u, u) \leq \mathcal{B}(u, u),
$$

$u=0$ is the only solution of

$$
\forall v \in H, \quad \mathcal{L}(u, v)=\mathcal{S}(u, v)
$$

Variants of Theorem 2 and Proposition 3 can be written, in which the operators belonging to $\mathcal{F}(X, V)$ satisfy a one-sided condition only.

THEOREM 4. Let $L: \operatorname{dom} L \subset H \cap X \rightarrow V$ be a linear Fredholm operator of index zero and $B: X \rightarrow V$ a linear $L$-compact operator. Consider the set $\mathcal{F}(X, V)$ of operators $S: X \rightarrow V$ such that for all $u \in X \cap V^{\prime}$

$$
\langle S u, u\rangle \leq\langle B u, u\rangle .
$$


Let $N: X \rightarrow V$ be a $L$-completely continuous operator. Assume that : for every $K>0$, there exists $M>0$ such that every solution $u$ of

$$
L u \cdot \lambda N u+(1-\lambda) B u
$$

with $\|u\|_{H}<K$ and $\lambda \in|0,1|$, verifies $\|u\|_{x}<M$.

If moreover,

(i) there exists a positive definite bilinear form $\mathcal{D}(.,$.$) such that, for all u \in$ dom $L$,

$$
\langle L u, u\rangle-\langle B u, u\rangle \geq \mathcal{D}(u, u)
$$

(ii) the operator $N$ admits the decomposition

$$
N(u)=G(u)+Q(u)
$$

where $G \in \mathcal{F}(X, V)$ and there exists $R>0$ such that, for all $u \in \operatorname{dom} L$ with $\|u\|_{H} \geq R$,

$$
\langle Q(u), u\rangle<\mathcal{D}(u, u) .
$$

Then there exists at least one solution $u$ of

$$
L u=N u
$$

The proof is similar to that of Theorem 2 .

REMARK. Notice that the linearity and the symmetry of the operators of $\mathcal{F}(X, V)$ are not required.

The following proposition is proved in C. De Coster, C. Fabry and P. Habets [4].

PROPOSITION 5. Let $H$ be a vector space and $\mathcal{L}, \mathcal{B}, \mathcal{D}$ be real, bilinear, symmetric forms on

$H$. Assume that

(i) there is some $m \in \mathbb{R}$ such that

$$
\mathcal{C}=\mathcal{L}-\mathcal{B}+m \mathcal{D}
$$

is a scalar product which makes $H$ a Hilbert space;

(ii) $\mathcal{D}$ is positive definite;

(iii) for any sequence $\left(u_{k}\right)_{k}$ such that $u_{k} \stackrel{\mathcal{C}}{\rightarrow} u$, one has

$$
\mathcal{D}\left(u_{k}, u_{k}\right) \rightarrow \mathcal{D}(u, u)
$$

Then the following equivalences hold :

(a) the eigenvalues $\lambda$ of the problem

$$
\forall v \in H, \mathcal{L}(u, v)-\mathcal{B}(u, v)=\lambda \mathcal{D}(u, v)
$$

are all strictly positive;

(b) there exists $\delta>0$ such that for any $u \in H$, one has

$$
(\mathcal{L}-\mathcal{B})(u, u) \geq \delta \mathcal{C}(u, u)
$$

(c) for any real bilinear symmetric form $\mathcal{S}$ on $H$ such that $\mathcal{S} \leq \mathcal{B}, u=0$ is the only solution of

$$
\forall v \in H, \mathcal{L}(u, v)=\mathcal{S}(u, v)
$$

\section{SYMMETRIC CASE}

In this section we will study the problem

$$
\begin{gathered}
u^{(4)}=f\left(t, u, u^{\prime}, u^{\prime \prime}, u^{\prime \prime \prime}\right), \\
u(0)=u(2 \pi), u^{\prime}(0)=u^{\prime}(2 \pi), u^{\prime \prime}(0)=u^{\prime \prime}(2 \pi), u^{\prime \prime \prime}(0)=u^{\prime \prime \prime}(2 \pi),
\end{gathered}
$$

under the general assumption that $f$ admits a decomposition of the form 


$$
f\left(t, u, u^{\prime}, u^{\prime \prime}, u^{\prime \prime \prime}\right)-\left(g\left(t, u, u^{\prime}\right) u^{\prime}\right)^{\prime}+h\left(t, u, u^{\prime}, u^{\prime \prime}, u^{\prime \prime \prime}\right) u+r\left(t, u, u^{\prime}, u^{\prime \prime}, u^{\prime \prime \prime}\right) \text {. }
$$

We will consider separately the case where the function $f$ stays asymptotically between two eigenlines and the case where it stays asymptotically at the left of all eigenlines.

\subsection{BETWEEN TIVO EI(IFNLINES}

THEOREM 6. Assume: that $\int$ satisfies (3.3) with

(III)(i) the functions

$$
h, r:[0,2 \pi] \times \mathbb{R}^{4} \rightarrow \mathbb{R}:(t, X) \rightarrow h(t, X), r(t, X),
$$

are measurable in $t \in[0,2 \pi]$ and contimuous in $X \in \mathbb{R}^{4}$

(ii) the function $g:[0,2 \pi] \times \mathbb{R}^{2} \rightarrow \mathbb{R}:(t, x, y) \rightarrow g(t, x, y)$ is differentiable and the partial derivatives are such that $\partial g / \partial t, \partial g / \partial x$ satisfy $L^{1}$-Carathéodory conditions, $\partial g / \partial y$ satisfy $L^{2}$ Carathéodory conditions and for all $(x, y) \in \mathbb{R}^{2}, g(0, x, y)=g(2 \pi, x, y)$;

(H2) there exist $m_{0}, m_{1}, m_{2} \in L^{1}(0,2 \pi), m_{3} \in L^{2}(0,2 \pi)$ and $\varepsilon>0$ such that, for all $X=$ $(x, y, z, w) \in \mathbb{R}^{4}$ and almost every $t \in[0,2 \pi]$,

$$
|r(t, X)| \leq m_{0}(t)+m_{1}(t)|x|^{1-\varepsilon}+m_{2}(t)|y|^{1-\varepsilon}+m_{3}(t)|z|^{1-\varepsilon}
$$

(II3)there exist $a_{1}, b_{1} \in L^{1}(0,2 \pi), a_{2}, b_{2} \in W^{1,1}(0,2 \pi)$ such that $a_{2}(0)=a_{2}(2 \pi), b_{2}(0)=b_{2}(2 \pi)$ and for all $x, y, z, w \in \mathbb{R}$ and for almost every $t \in[0,2 \pi]$,

$$
\begin{gathered}
a_{1}(t) \leq h(t, x, y, z, w) \leq b_{1}(t), \\
b_{2}(t) \leq g(t, x, y) \leq a_{2}(t) .
\end{gathered}
$$

If moreover,

(F) there exist $\delta>0$ and a decomposition $H_{2 \pi}^{2}=\bar{H} \oplus \tilde{H}$ with $\operatorname{dim} \bar{H}<\infty$ such that, for any $\bar{u} \in \bar{H}, \tilde{u} \in \tilde{H}$,

$$
\int_{0}^{2 \pi}\left[\tilde{u}^{\prime \prime 2}+b_{2} \tilde{u}^{\prime 2}-b_{1} \tilde{u}^{2}\right]-\int_{0}^{2 \pi}\left[\bar{u}^{\prime \prime 2}+a_{2} \bar{u}^{\prime 2}-a_{1} \bar{u}^{2}\right] \geq \delta\|u\|_{H^{2}}^{2},
$$

then the problem (3.1)-(3.2) has at least one solution.

PROOF. We will apply Theorem 2 with the spaces $H:=H_{2 \pi}^{2}, V:=L^{1}, X:=H_{2 \pi}^{3}$ and the operators

$$
\begin{aligned}
& L \quad: \quad \operatorname{dom} L=\left\{u \in W^{4,1}: u \text { satisfies }(3.2)\right\} \rightarrow L^{1}: u \rightarrow u^{(4)} \\
& A: H_{2 \pi}^{3} \rightarrow L^{1}: u \rightarrow\left(a_{2} u^{\prime}\right)^{\prime}+a_{1} u \\
& B \quad: \quad H_{2 \pi}^{3} \rightarrow L^{1}: u \rightarrow\left(b_{2} u^{\prime}\right)^{\prime}+b_{1} u \\
& G: H_{2 \pi}^{3} \rightarrow \mathcal{L}\left(H_{2 \pi}^{3}, L^{1}\right): x \rightarrow G(x) \text { defined by } G(x) u=\left(g\left(t, x, x^{\prime}\right) u^{\prime}\right)^{\prime}+h\left(t, x, x^{\prime}, x^{\prime \prime}, x^{\prime \prime \prime}\right) u \\
& Q: H_{2 \pi}^{3} \rightarrow L^{1}: u \rightarrow r\left(t, u, u^{\prime}, u^{\prime \prime}, u^{\prime \prime \prime}\right) \\
& N \quad: \quad H_{2 \pi}^{3} \rightarrow L^{1}: u \rightarrow G(u) u+Q(u) .
\end{aligned}
$$

It is easy to see that $L$ is a Fredholm operator of index zero and has a compact generalized inverse from $L^{1}$ into $H_{2 \pi}^{3}$. It is not difficult to prove that $A, B$ are $L$-compact and $N: H_{2 \pi}^{3} \rightarrow L^{1}$ will be $L$-completely continuous if we prove that it is continuous and maps bounded sets into bounded sets. For that purpose, we will use the following result :

Let $X$ be a metric space, $\left(f_{n}\right)$ a sequence in $X$ and $f \in X$ be such that for any subsequence of $\left(f_{n}\right)$ there exists a sub-subsequence which converges to $f$. Then, the initial sequence converges to $f$.

So, let $\left(u_{n}\right) \subset H_{2 \pi}^{3}$ be such that $u_{n}$ converges to some $u$ in the $H_{2 \pi}^{3}$-norm, and let $\left(u_{n_{k}}\right)$ be a subsequence of $\left(u_{n}\right)$. Consider the sequence $\varphi_{n}(t)=f\left(t, u_{n}(t), u_{n}^{\prime}(t), u_{n}^{\prime \prime}(t), u_{n}^{\prime \prime \prime}(t)\right)$ and the subsequence $\left(\varphi_{n_{k}}\right)$ which corresponds to $\left(u_{n_{k}}\right)$. As $\left(u_{n_{k}}\right)$ converges to $u$ in the $H_{2 \pi}^{3}$-norm, there exists a 
sub-sulsecquence $\left(u_{n_{k}}\right)$ of $\left(u_{n_{k}}\right)$ such that for $i=0,1,2,3$,

(i) $u_{n_{k}}^{(1)}(t) \rightarrow u^{(2)}(t)$, a.e. on $[0,2 \pi]$;

(ii) there exists $h \in L^{2}(0,2 \pi)$ such that $\left|u_{n_{k},}^{(z)}(t)\right| \leq h(t)$ on $[0,2 \pi]$ (see, for example, Brezis [3, p. $58 \mid)$.

By hypothesis (II), we have $\varphi_{n_{k}}(t) \rightarrow \varphi(t)$ a.e. on $[0,2 \pi]$, where $\varphi(t)=f\left(t, u(t), u^{\prime}(t), u^{\prime \prime}(t), u^{\prime \prime \prime}(t)\right)$. Moreover, from the $I^{3}$-bound on the sub-subsequence $\left(u_{n_{k},}\right)$ we deduce a $\mathcal{C}^{2}$-bound on it, and, by (HI), (H2) and (II3), we can find a function $g \in L^{1}$ such that $\left|\varphi_{n_{k},}(t)\right| \leq g(t)$. The continuity of $N$ follows from the Lebesgue dominated convergence theorem. Moreover, we easily deduce from the structure of $N$ that it maps bounded sets into bounded sets.

Now we will prove that hypothesis (c) of Theorem 2 is satisfied. By the continuous injection of $H^{2}$ into $\mathcal{C}^{1}$ and the hypothesis on $f$, we have that, if there exists $K>0$ such that for all solution $u$ of (2.3) we have $\|u\|_{H^{2}}<K$, then there exists $M_{1}=M_{1}(K)$ such that $\left\|u^{(4)}\right\|_{L^{1}} \leq M_{1}$. It is easy to conclude that there exists $M_{2}$ such that $\|u\|_{H^{3}} \leq M_{2}$.

The rest of the proof is left to the reader.

The following lemma is necessary in order to apply Proposition 3.

LEMMA 7. Assume that there exist $a_{1}, b_{1} \in L^{1}(0,2 \pi), a_{2}, b_{2} \in W^{1,1}(0,2 \pi)$ with $a_{2}(0)$ $a_{2}(2 \pi), b_{2}(0)=b_{2}(2 \pi)$ such that $a_{1}(t) \lesssim b_{1}(t)$ and $a_{1}(t)+b_{2}(t)<a_{2}(t)+b_{1}(t)$ for a.e. $t \in[0,2 \pi]$. Then, there exists $n \in \mathbb{N}^{*}$ such that, for all $u \in H_{2 \pi}^{2}$,

$$
\int_{0}^{2 \pi}\left[u^{\prime \prime 2}-\left(n\left(b_{2}-a_{2}\right)-a_{2}\right) u^{2}+\left(n\left(b_{1}-a_{1}\right)-a_{1}\right) u^{2}\right] \geq \frac{1}{2}\|u\|_{H^{2}}^{2} .
$$

PROOF. Assume by contradiction that there exists a sequence $\left(u_{n}\right) \subset H_{2 \pi}^{2}$ such that $\left\|u_{n}\right\|_{H^{2}}=$ 1 and

$$
\int_{0}^{2 \pi}\left[u_{n}^{\prime 2}-\left(n\left(b_{2}-a_{2}\right)-a_{2}\right) u_{n}^{\prime 2}+\left(n\left(b_{1}-a_{1}\right)-a_{1}\right) u_{n}^{2}\right]<\frac{1}{2} .
$$

As $a_{2}(t) \geq b_{2}(t)$ and $b_{1}(t) \geq a_{1}(t)$ on $[0,2 \pi]$, we can deduce from this relation that there exists $\beta>0$ such that, for all $n$,

i.e.

$$
1=\left\|u_{n}\right\|_{H^{2}}^{2} \leq \beta\left\|u_{n}\right\|_{\mathcal{C}^{1}}^{2}+\frac{1}{2}
$$

$$
\left\|u_{n}\right\|_{\mathcal{C}^{1}}^{2} \geq \frac{1}{2 \beta}
$$

On the other hand, we can find a subsequence, still denoted $\left(u_{n}\right)$, such that $\left(u_{n}\right)$ converges to some $u$ in $\mathcal{C}^{1}$. We then have,

$$
\begin{aligned}
0 \leq \int_{0}^{2 \pi}\left[\left(a_{2}-b_{2}\right) u^{2}+\left(b_{1}-a_{1}\right) u^{2}\right] & =\lim _{n \rightarrow \infty} \int_{0}^{2 \pi}\left[\left(a_{2}-b_{2}\right) u_{n}^{\prime 2}+\left(b_{1}-a_{1}\right) u_{n}^{2}\right] \\
& \leq \lim _{n \rightarrow \infty} \frac{1}{n}\left[\frac{1}{2}-\int_{0}^{2 \pi}\left[u_{n}^{\prime 2}+a_{2} u_{n}^{\prime 2}-a_{1} u_{n}^{2}\right]\right]=0 .
\end{aligned}
$$

We deduce from the hypothesis $a_{1}(t)+b_{2}(t)<a_{2}(t)+b_{1}(t)$ for a.e. $t \in[0,2 \pi]$ with $a_{1}(t) \lesssim b_{1}(t)$, that $u \equiv 0$, which contradicts (3.5).

Using this, we will be able to give some simple hypothesis which ensure that hypothesis $(F)$ of Theorem 6 is satisfied.

COROLLARY 8. Assume that $f$ satisfies (3.3), hypotheses (H1) to (H3) of Theorem 6 and the hypotheses of Lemma 7. If moreover, there exist $\alpha_{1}, \beta_{1}, \alpha_{2}, \beta_{2} \in \mathbb{R}$ such that for a.e. $t \in[0,2 \pi]$,

$$
\begin{gathered}
\alpha_{1} \lesssim a_{1}(t) \leq b_{1}(t) \lesssim \beta_{1}, \\
\beta_{2} \leq b_{2}(t) \leq a_{2}(t) \leq \alpha_{2}
\end{gathered}
$$

and for all $k \in \mathbb{N}$, for all $\lambda \in(0,1)$

$$
\left(\lambda \beta_{1}+(1-\lambda) \alpha_{1}\right)-\left(\lambda \beta_{2}+(1-\lambda) \alpha_{2}\right) k^{2} \neq k^{4}
$$


then problem (3.1)-(3.2) has at least one solution.

REMARK. It is easy to see that the last conditions of Corollary 8 mean that the point $(g(t, x, y), h(t, x, y, z, w))$ always lies in a rectangle which does not intersect any eigenline.

PROOF. Using Lemma 7, we can apply Proposition 3 with $H=H_{2 \pi}^{2}$, the bilinear forms $\mathcal{L}, \mathcal{A}, \mathcal{B}: H \times H \rightarrow \mathbb{R}$ being defined respectively by

$$
\mathcal{L}(u, v)=\int_{0}^{2 \pi} u^{\prime \prime} v^{\prime \prime}, \quad \mathcal{A}(u, v)=\int_{0}^{2 \pi}\left[-a_{2} u^{\prime} v^{\prime}+a_{1} u v\right], \quad \mathcal{B}(u, v)=\int_{0}^{2 \pi}\left[-b_{2} u^{\prime} v^{\prime}+b_{1} u v\right] .
$$

So condition $(F)$ holds if we can prove that the interval $[0,1]$ does not contain eigenvalues $\lambda$ of the problem

$$
\begin{gathered}
u^{(4)}=\left(\left(\lambda b_{2}+(1-\lambda) a_{2}\right) u^{\prime}\right)^{\prime}+\left(\lambda b_{1}+(1-\lambda) a_{1}\right) u, \\
u(0)=u(2 \pi), u^{\prime}(0)=u^{\prime}(2 \pi), u^{\prime \prime}(0)=u^{\prime \prime}(2 \pi), u^{\prime \prime \prime}(0)=u^{\prime \prime \prime}(2 \pi) .
\end{gathered}
$$

Assume by contradiction that there exist $\lambda \in[0,1]$ and $u \neq 0$ solution of (3.7)-(3.8). As $\alpha_{1} \lesssim \lambda b_{1}(t)+(1-\lambda) a_{1}(t) \lesssim \beta_{1}$ on $[0,2 \pi]$, we have, by the variational characterization of the eigenvalues, that the eigenvalues $\mu_{2}, \nu_{2}, \rho_{2}$ of the problems

$$
\begin{aligned}
& u^{(4)}-\alpha_{2} u^{\prime \prime}-\alpha_{1} u=\mu u \\
& u^{(4)}-\left(\left(\lambda b_{2}+(1-\lambda) a_{2}\right) u^{\prime}\right)^{\prime}-\left(\lambda b_{1}+(1-\lambda) a_{1}\right) u=\nu u, \\
& u^{(4)}-\beta_{2} u^{\prime \prime}-\beta_{1} u=\rho u
\end{aligned}
$$

with boundary conditions (3.8), satisfy

$$
\rho_{\imath}<\nu_{\imath}<\mu_{\imath} \text {, for all } \imath \in \mathbb{N},
$$

assuming the eigenvalues of the three problems to be arranged by increasing order.

As the problem (3.7)-(3.8) is assumed to have a nontrivial solution, there must be some $i \in \mathbb{N}$ such that $\nu_{2}=0$. By continuity of the eigenvalue, there exists $\left.\theta \in\right] 0,1[$ such that the problem

$$
\begin{gathered}
u^{(4)}-\left(\theta \beta_{2}+(1-\theta) \alpha_{2}\right) u^{\prime \prime}-\left(\theta \beta_{1}+(1-\theta) \alpha_{1}\right) u=0, \\
u(0)=u(2 \pi), u^{\prime}(0)=u^{\prime}(2 \pi), u^{\prime \prime}(0)=u^{\prime \prime}(2 \pi), u^{\prime \prime \prime}(0)=u^{\prime \prime \prime}(2 \pi),
\end{gathered}
$$

has a nontrivial solution, which, according to Lemma 1, contradicts the hypothesis (3.6).

REMARK. Letting $q=\left(\alpha_{2}+\beta_{2}\right) / 2, b=\left(\alpha_{2}-\beta_{2}\right) / 2, p=\left(\alpha_{1}+\beta_{1}\right) / 2$, and $a=\left(\beta_{1}-\alpha_{1}\right) / 2$, the condition (3.6) can be written equivalently as : for all $k \in \mathbb{N}$

$$
k^{4}+q k^{2}-p \neq 0
$$

and

\subsection{AT THE LEFT OF ALL EIGENLINES}

$$
1 \geq \frac{a}{\left|k^{4}+q k^{2}-p\right|}+\frac{b k^{2}}{\left|k^{4}+q k^{2}-p\right|} .
$$

In this subsection, we will consider problem (3.1)-(3.2) with $f$ as in (3.3), but we will assume only one-sided conditions on $h$ and $g$. Unfortunately, in that case we cannot have a $u^{\prime \prime \prime}$-dependence in $f$. This is due to the fact that we cannot find an $H^{3}$ bound on the solutions of (2.3) from an $H^{2}$-bound, as in Theorem 6 .

The proofs of the results of this subsection follow from Theorem 4 and Proposition 5. We take here $H=X=H_{2 \pi}^{2}$.

THEOREM 9. Let $f:[0,2 \pi] \times \mathbb{R}^{3} \rightarrow \mathbb{R}$ be a function which satisfies the hypothesis (H1) and (H2) of Theorem 6 with $h, r:[0,2 \pi] \times \mathbb{R}^{3} \rightarrow \mathbb{R}$. Assume 
(1I3') there exist $b_{1} \in I^{\prime}(0,2 \pi), b_{2} \in \mathbb{H}^{1,1}(0,2 \pi)$ such that $b_{2}(0) \quad b_{2}(2 \pi)$ and for all $(x, y, z) \in \mathbb{R}^{3}$ and almost every $t \in[0,2 \pi]$.

$$
\begin{gathered}
h(t, x, y, z) \leq b_{1}(t), \\
b_{2}(t) \leq g(t, x, y) .
\end{gathered}
$$

(II4) there exist $d:[0,2 \pi] \times \mathbb{R}^{2} \rightarrow \mathbb{R}$, a $L^{2}$-Carathéodory function and $c \in L^{1}(0,2 \pi)$ such that, for all $(u, v, w) \in \mathbb{R}^{3}$ and a.e. $t \in[0,2 \pi]$,

$$
|f(t, u, v, w)| \leq d(t, u, v)|w|+c(t) .
$$

If moreover, there exists $\delta>0$ such that, for all $u \in \operatorname{dom} L$,

$$
\int_{0}^{2 \pi}\left[u^{\prime 2}+b_{2} u^{\prime 2}-b_{1} u^{2}\right] \geq \delta\|u\|_{H^{2}}^{2}
$$

then the problem

$$
\begin{gathered}
u^{(4)}=f\left(t, u, u^{\prime}, u^{\prime \prime}\right), \\
u(0)=u(2 \pi), u^{\prime}(0)=u^{\prime}(2 \pi), u^{\prime \prime}(0)=u^{\prime \prime}(2 \pi), u^{\prime \prime \prime}(0)=u^{\prime \prime \prime}(2 \pi),
\end{gathered}
$$

with

$$
f\left(t, u, u^{\prime}, u^{\prime \prime}\right)=\left(g\left(t, u, u^{\prime}\right) u^{\prime}\right)^{\prime}+h\left(t, u, u^{\prime}, u^{\prime \prime}\right) u+r\left(t, u, u^{\prime}, u^{\prime \prime}\right)
$$

has at least one solution.

As in the previous case we will give some simple hypotheses which ensure that condition (3.9) is satisfied.

COROLLARY 10. Assume that $f$ is a $L^{1}$-Carathéodory function which satisfies (3.12) and (H1) to (H4) of Theorem 9. If moreover there exist $\beta_{1}, \beta_{2} \in \mathbb{R}$ such that, for almost every $t \in[0,2 \pi]$,

$$
\beta_{2} \leq b_{2}(t), \quad b_{1}(t) \lesssim \beta_{1},
$$

and, for all $k \in \mathbb{N}$, for all $\lambda<0$,

$$
\left(\beta_{1}+\lambda\right)-\left(\beta_{2}-\lambda\right) k^{2} \neq k^{4}
$$

then the problem (3.10)-(3.11) has at least one solution.

REMARK. We can observe that condition (3.13) is equivalent to : for all $k \in \mathbb{N}$

$$
0 \leq k^{4}+\beta_{2} k^{2}-\beta_{1}
$$

\section{NON-SYMMETRIC CASE}

In the previous section, we have assumed that the function $f$ admits a symmetric decomposition of the form (3.3). It does not seem easy to give practical hypothesis which ensure that such a decomposition exists. On the opposite, it is easy to find practical conditions under which $f$ has a decomposition of the form

$$
f\left(t, u, u^{\prime}, u^{\prime \prime}\right)=f_{2}\left(t, u, u^{\prime}, u^{\prime \prime}\right) u^{\prime \prime}+f_{1}\left(t, u, u^{\prime}, u^{\prime \prime}\right) u^{\prime}+f_{0}\left(t, u, u^{\prime}, u^{\prime \prime}\right) u+r\left(t, u, u^{\prime}, u^{\prime \prime}\right)
$$

with $r$ bounded. For a given function $u$, the operator $v \rightarrow f_{2}\left(., u(),. u^{\prime}(),. u^{\prime \prime}().\right) v^{\prime \prime}$ is not symmetric; that difficulty is dealt with below by treating that operator as a "perturbation" of a linear symmetric operator. For the sake of simplicity, we will assume that $f_{1} \equiv 0$, since the presence of the corresponding term introduce only technical difficulties. As in section 3 , we will consider separately the case where the function $f$ stays asymptotically between two eigenlines and the case where it stays asymptotically at the left of all eigenlines. 


\subsection{BETWEEN TWO EIGENIJINES}

THEOREM 11. Let $f:\left[0,2 \pi \mid \times \mathbb{R}^{3} \rightarrow \mathbb{R}:(t, X) \rightarrow f(t, X)\right.$ be measurable in $t \in[0,2 \pi]$ for all $X \in \mathbb{R}^{3}$ and continuous in $X \in \mathbb{R}^{3}$ for a.e. $t \in[0,2 \pi]$. Assume that there exists $p, q \in \mathbb{R}, a>0$, $b \geq 0, \alpha, \gamma \in L^{1}\left((0,2 \pi), \mathbb{R}^{\dagger}\right)$ such that for all $(u, v, u) \in \mathbb{R}^{3}$ and a.e. $t \in[0,2 \pi]$,

(i) $|f(t, u, v, w)-p u-q w| \leq \alpha(t)|u|+b|w|+\gamma(t)$;

(ii) $\alpha(t)<a$;

(iii) there exists $\xi>b / 2$ such that for all $k \in \mathbb{N}$

$$
\begin{gathered}
k^{4}+q k^{2}-p \neq 0, \\
1 \geq \frac{a}{\left|k^{4}+q k^{2}-p\right|}+\frac{b\left(\left(k^{4} / 2 \xi\right)+(\xi / 2)\right)}{\left|k^{4}+q k^{2}-p\right|} .
\end{gathered}
$$

Then, there exists at least one solution $u$ of

$$
\begin{gathered}
u^{(4)}=f\left(t, u, u^{\prime}, u^{\prime \prime}\right), \\
u(0)=u(2 \pi), u^{\prime}(0)=u^{\prime}(2 \pi), u^{\prime \prime}(0)=u^{\prime \prime}(2 \pi), u^{\prime \prime \prime}(0)=u^{\prime \prime \prime}(2 \pi) .
\end{gathered}
$$

PROOF. Let us show first that $f$ admits a decomposition of the form

$$
f(t, u, v, w)=g(t, u, v, w) u+h(t, u, v, w) w+r(t, u, v, w),
$$

with

$$
|g(t, u, v, w)-p| \leq \alpha(t), \quad|h(t, u, v, w)-q| \leq b, \quad|r(t, u, v, w)| \leq \gamma(t) .
$$

Let $m(t, u, w)=\alpha(t)|u|+b|w|+\gamma(t)$. If $m(t, u, w)=0$, we have $f(t, u, v, w)=p u+q w$. Otherwise, we can write the relation

$$
f(t, u, v, w)-p u-q w=\frac{f(t, u, v, w)-p u-q w}{m(t, u, w)}[(\alpha(t) \operatorname{sgn} u) u+(b \operatorname{sgn} w) w+\gamma(t)],
$$

from which the required result is easily deduced.

We will apply Theorem 2 with $H=X=H_{2 \pi}^{2}, V=L^{1}$,

$$
\begin{array}{ll}
L: & \operatorname{dom} L=\left\{u \in W^{4,1}: u \text { satisfies }(4.4)\right\} \rightarrow L^{1}: u \rightarrow u^{(4)} \\
A: & H_{2 \pi}^{2} \rightarrow L^{1}: u \rightarrow(p-\alpha(t)) u+q u^{\prime \prime} \\
B: & H_{2 \pi}^{2} \rightarrow L^{1}: u \rightarrow(p+\alpha(t)) u+q u^{\prime \prime} \\
G: & H_{2 \pi}^{2} \rightarrow \mathcal{L}\left(H_{2 \pi}^{2}, L^{1}\right): x \rightarrow G(x) \text { defined by } G(x) u=q u^{\prime \prime}+g\left(t, x, x^{\prime}, x^{\prime \prime}\right) u \\
Q: & H_{2 \pi}^{2} \rightarrow L^{1}: u \rightarrow\left(h\left(t, u, u^{\prime}, u^{\prime \prime}\right)-q\right) u^{\prime \prime}+r\left(t, u, u^{\prime}, u^{\prime \prime}\right) .
\end{array}
$$

Let us prove that, for some $\varepsilon>0$, the hypothesis (i) and (ii) of Theorem 2 will be satisfied with

$$
\mathcal{D}(u, u)=\left(\varepsilon+\frac{b}{2 \xi}\right) \int_{0}^{2 \pi} u^{\prime 2}+\left(\varepsilon+\frac{\xi b}{2}\right) \int_{0}^{2 \pi} u^{2}
$$

and $\bar{H}, \tilde{H}$ defined in the following way. Let

$$
\bar{K}=\left\{k \in \mathbb{N}: k^{4}+q k^{2}-p<0\right\}, \quad \tilde{K}=\left\{k \in \mathbb{N}: k^{4}+q k^{2}-p>0\right\}
$$

and consider the decomposition $H_{2 \pi}^{2}=\bar{H} \oplus \tilde{H}$ where

$$
\begin{aligned}
& \bar{H}=\left\{\bar{u}=\sum_{k \in \bar{K}}\left(a_{k} \sin k t+b_{k} \cos k t\right) \mid a_{k}, b_{k} \in \mathbb{R}\right\}, \\
& \tilde{H}=\left\{\tilde{u}=\sum_{k \in \tilde{K}}\left(a_{k} \sin k t+b_{k} \cos k t\right) \mid a_{k}, b_{k} \in \mathbb{R}\right\} .
\end{aligned}
$$


Let us prove hypothesis (i) of 'Theorem 2. If $u \in H_{2 \pi}^{2}$, it can be written under the form

$$
u(t)=\sum_{k=0}^{\infty}\left(a_{k} \sin k t+b_{k} \cos k t\right) \text {. }
$$

We then have, for $u \neq 0$.

$$
\begin{aligned}
\int_{0}^{2 \pi}\left[\tilde{u}^{\prime 2}\right. & \left.+q \tilde{u}^{\prime 2}-(p+\alpha(t)) \tilde{u}^{2}\right]-\int_{0}^{2 \pi}\left[\bar{u}^{\prime 2}+q \bar{u}^{\prime 2}-(p-\alpha(t)) \bar{u}^{2}\right] \\
& >\int_{0}^{2 \pi}\left[\tilde{u}^{\prime \prime 2}+q \tilde{u}^{\prime 2}-(p+a) \tilde{u}^{2}\right]-\int_{0}^{2 \pi}\left[\bar{u}^{\prime 2}+q \bar{u}^{\prime 2}-(p-a) \bar{u}^{2}\right] \\
& >\pi\left[\sum_{k \in \tilde{K}}\left[k^{4}+q k^{2}-(p+a)\right]\left(a_{k}^{2}+b_{k}^{2}\right)-\sum_{k \in \bar{K}}\left[k^{4}+q k^{2}-(p-a)\right]\left(a_{k}^{2}+b_{k}^{2}\right)\right] \\
& >\pi\left[\frac{b}{2 \xi} \sum_{k \in \mathrm{N}} k^{4}\left(a_{k}^{2}+b_{k}^{2}\right)+\frac{\xi b}{2} \sum_{k \in \mathrm{N}}\left(a_{k}^{2}+b_{k}^{2}\right)\right] \\
& >\frac{b}{2 \xi} \int_{0}^{2 \pi} u^{\prime \prime 2}+\frac{\xi b}{2} \int_{0}^{2 \pi} u^{2},
\end{aligned}
$$

where we have used the hypothesis $\alpha(t)<a$ and the inequalities

$$
\begin{aligned}
& \forall k \in \bar{K},\left(1+\frac{b}{2 \xi}\right) k^{4}+q k^{2}-\left(p-a-\frac{b \xi}{2}\right) \leq 0, \\
& \forall k \in \tilde{K},\left(1-\frac{b}{2 \xi}\right) k^{4}+q k^{2}-\left(p+a+\frac{b \xi}{2}\right) \geq 0,
\end{aligned}
$$

which are satisfied if (4.1)-(4.2) are satisfied. Now let us prove that there exists $\varepsilon>0$ such that

$\int_{0}^{2 \pi}\left[\tilde{u}^{\prime \prime 2}+q \tilde{u}^{\prime 2}-(p+\alpha(t)) \tilde{u}^{2}\right]-\int_{0}^{2 \pi}\left[\bar{u}^{\prime \prime 2}+q \bar{u}^{\prime 2}-(p-\alpha(t)) \bar{u}^{2}\right] \geq \frac{b}{2 \xi} \int_{0}^{2 \pi} u^{\prime 2}+\frac{\xi b}{2} \int_{0}^{2 \pi} u^{2}+\varepsilon\|u\|_{H^{2}}^{2}$.

Otherwise, for all $n$, there exists $u_{n} \in H_{2 \pi}^{2}$ with $\left\|u_{n}\right\|_{H^{2}}=1$ and

$0<\int_{0}^{2 \pi}\left[\tilde{u}_{n}^{\prime \prime 2}+q \tilde{u}_{n}^{\prime 2}-(p+\alpha(t)) \tilde{u}_{n}^{2}\right]-\int_{0}^{2 \pi}\left[\bar{u}_{n}^{\prime \prime 2}+q \bar{u}_{n}^{\prime 2}-(p-\alpha(t)) \bar{u}_{n}^{2}\right]-\left[\frac{b}{2 \xi} \int_{0}^{2 \pi} u_{n}^{\prime \prime 2}+\frac{\xi b}{2} \int_{0}^{2 \pi} u_{n}^{2}\right]<1 / n$.

By going if necessary to subsequences, as $\left\|u_{n}\right\|_{H^{2}}=1$, we can assume that $u_{n}$ converges weakly in $H^{2}$ and in the $\mathcal{C}^{1}$-norm to some $u, \bar{u}_{n}$ converges to $\bar{u}$ in the $H^{2}$-norm and

$$
\begin{aligned}
\lim _{n \rightarrow \infty}\left(1-\frac{b}{2 \xi}\right) \int_{0}^{2 \pi} \tilde{u}_{n}^{\prime 2}= & -\int_{0}^{2 \pi}\left[q \tilde{u}^{\prime 2}-\left(p+\alpha(t)+\frac{\xi b}{2}\right) \tilde{u}^{2}\right] \\
& +\int_{0}^{2 \pi}\left[\left(1+\frac{b}{2 \xi}\right) \bar{u}^{\prime 2}+q \bar{u}^{\prime 2}-\left(p-\alpha(t)-\frac{\xi b}{2}\right) \bar{u}^{2}\right] .
\end{aligned}
$$

By (4.5), (4.6) and the weak lower semi-continuity of the norm we have $u \equiv 0$. Then we know that $u_{n}$ converges weakly in $H^{2}$ and in the $\mathcal{C}^{1}$-norm to 0 and that $\bar{u}_{n}$ converges to 0 in the $H^{2}$-norm. By (4.6), we see that $\tilde{u}_{n}^{\prime \prime}$ converges to 0 in the $L^{2}$-norm and then we have that $u_{n}$ converges to 0 in the $H^{2}$-norm, which contradicts the fact that $\left\|u_{n}\right\|_{H^{2}}=1$. Consequently, hypothesis (i) of Theorem 2 is satisfied.

Now we will prove hypothesis (ii) of Theorem 2 i.e. there exists $R>0$ such that for all $u=\bar{u}+\tilde{u}$ with $\bar{u} \in \bar{H}, \tilde{u} \in \tilde{H}$ and $\|u\|_{H^{2}}>R$ we have

$$
\int_{0}^{2 \pi}\left[\left(h\left(t, u, u^{\prime}, u^{\prime \prime}\right)-q\right) u^{\prime \prime}+r\left(t, u, u^{\prime}, u^{\prime \prime}\right)\right](\tilde{u}-\bar{u})<\left(\frac{b}{2 \xi}+\varepsilon\right) \int_{0}^{2 \pi} u^{\prime 2}+\left(\frac{\xi b}{2}+\varepsilon\right) \int_{0}^{2 \pi} u^{2} .
$$

In fact, we have, for some constant $c>0$,

$$
\begin{aligned}
\int_{0}^{2 \pi}\left(h\left(t, u, u^{\prime}, u^{\prime \prime}\right)-q\right) u^{\prime \prime}(\tilde{u}-\bar{u}) & +\int_{0}^{2 \pi} r\left(t, u, u^{\prime}, u^{\prime \prime}\right)(\tilde{u}-\bar{u}) \\
& \leq \int_{0}^{2 \pi} b\left|u^{\prime \prime}\right||\tilde{u}-\bar{u}|+\int_{0}^{2 \pi} \gamma(t)|\tilde{u}-\bar{u}| \\
& \leq \frac{b}{2 \xi} \int_{0}^{2 \pi} u^{\prime \prime 2}+\frac{\xi b}{2} \int_{0}^{2 \pi} u^{2}+c\|\gamma\|_{L^{1}}\|u\|_{H^{2}} \\
& <\frac{b}{2 \xi} \int_{0}^{2 \pi} u^{\prime 2}+\frac{\xi b}{2} \int_{0}^{2 \pi} u^{2}+\varepsilon\|u\|_{H^{2}}^{2}
\end{aligned}
$$


for $\|u\|_{H^{2}} \geq R$, with $R$ big enough.

So we can apply Theorem 2 .

REMARK. 1) In the case where $a \equiv 0$, the result is still true provided that we replace (4.2) by a strict inequality.

2) In the case where $b=0$, we obtain the same conditions as in the symmetric case.

3) As the following example shows, the above result is not contained in the results of Del PinoManasevich [5]. Consider the case where $p=-2, q=-4, a=13 / 15$ and $b \in] 1 / 15,2 / 15]$. The existence of a solution can be proven by our theorem with $\xi=1$, whereas the results of Del Pino-Manasevich do not apply in that case.

\subsection{AT THE LEFT OF ALL EIGENLINES}

In this subsection, we come back to the situation where the nonlinearity can be considered to be asymptotically "at the left of all eigenlines", leading to one-sided existence conditions. We consider two different situations, depending on the regularity of the limiting functions.

THEOREM 12. Let $f:[0,2 \pi] \times \mathbb{R}^{3} \rightarrow \mathbb{R}$ be a function which satisfies hypothesis (H4) of Theorem 9. Assume that there exists $a \in \mathbb{R}, b \geq 0, q \in \mathbb{R}, \alpha, \gamma \in L^{1}\left((0,2 \pi), \mathbb{R}^{+}\right)$such that for all $(u, v, w) \in \mathbb{R}^{3}$ and a.e. $t \in[0,2 \pi]$,

(i) $(f(t, u, v, w)-q w) u \leq \alpha(t) u^{2}+b|u w|+\gamma(t)|u|$;

(ii) $\alpha(t) \lesssim a$;

(iii) there exists $\xi>b / 2$ such that for all $k \in \mathbb{N}$

$$
k^{4}+q k^{2} \geq a+b\left(\frac{k^{4}}{2 \xi}+\frac{\xi}{2}\right)
$$

Then, there exists at least one solution $u$ of (4.3)-(4.4).

PROOF. We apply Theorem 4 with $H=X=H_{2 \pi}^{2}, V=L^{1}$,

$$
\begin{aligned}
& L: \quad \operatorname{dom} L=\left\{u \in W^{4,1}: u \text { satisfies }(4.4)\right\} \rightarrow L^{1}: u \rightarrow u^{(4)}, \\
& B: H_{2 \pi}^{2} \rightarrow L^{1}: u \rightarrow \alpha(t) u+q u^{\prime \prime}, \\
& G: H_{2 \pi}^{2} \rightarrow L^{1}: u \rightarrow \alpha(t) u+q u^{\prime \prime} \\
& Q: \quad H_{2 \pi}^{2} \rightarrow L^{1}: u \rightarrow f\left(t, u, u^{\prime}, u^{\prime \prime}\right)-\left[\alpha(t) u+q u^{\prime \prime}\right] .
\end{aligned}
$$

The proof follows by arguments similar to those of Theorem 11 .

A slightly different result can be obtained, assuming more regularity on $\beta$.

THEOREM 13. Let $f:[0,2 \pi] \times \mathbb{R}^{3} \rightarrow \mathbb{R}$ be a function which satisfies hypothesis (H4) of Theorem 9. Assume that there exist $q, a \in \mathbb{R}, \alpha, \gamma \in L^{1}\left((0,2 \pi), \mathbb{R}^{+}\right), \beta \in \mathcal{C}^{2}(0,2 \pi)$ such that $\beta(0)=\beta(2 \pi), \beta^{\prime}(0)=\beta^{\prime}(2 \pi)$ and for all $(u, v, w) \in \mathbb{R}^{3}$ and a.e. $t \in[0,2 \pi]$,

(i) $(f(t, u, v, w)-q w) u \leq \alpha(t) u^{2}+\beta(t) u w+\gamma(t)|u|$;

(ii) $\alpha(t)<a, \beta(t) \geq 0, \beta^{\prime \prime}(t) \leq r$;

(iii) for all $k \in \mathbb{N}$

$$
k^{4}+q k^{2}-(a+r / 2) \geq 0 .
$$

Then, there exists at least one solution $u$ of (4.3)-(4.4).

PROOF. We apply Theorem 4 with $H=X=H_{2 \pi}^{2}, V=L^{1}$,

$$
\begin{aligned}
& L: \quad \operatorname{dom} L=\left\{u \in W^{4,1}: u \text { satisfies }(4.4)\right\} \rightarrow L^{1}: u \rightarrow u^{(4)}, \\
& B: H_{2 \pi}^{2} \rightarrow L^{1}: u \rightarrow \alpha(t) u+(\beta(t)+q) u^{\prime \prime}, \\
& G: H_{2 \pi}^{2} \rightarrow L^{1}: u \rightarrow f\left(t, u, u^{\prime}, u^{\prime \prime}\right)-\gamma(t) \operatorname{sgn} u, \\
& Q: \quad H_{2 \pi}^{2} \rightarrow L^{1}: u \rightarrow \gamma(t) \operatorname{sgn} u .
\end{aligned}
$$

The only significant difference in the proof of this theorem is the way the inequality

$$
\int_{0}^{2 \pi}\left[u^{(4)}-\alpha(t) u-(\beta(t)+q) u^{\prime \prime}\right] u>0
$$


is obtained. In this case, we use integration by parts. We obtain. for $u \neq 0$,

$$
\begin{aligned}
\int_{0}^{2 \pi}\left[u^{(4)}-\alpha(t) u-(\beta(t)+q) u^{\prime \prime}\right] u & =\int_{0}^{2 \pi}\left[u^{\prime \prime 2}+q u^{2}-\alpha(t) u^{2}-\beta(t) u u^{\prime \prime}\right] \\
& >\int_{0}^{2 \pi}\left[u^{\prime \prime 2}+q u^{\prime 2}-a u^{2}+\beta(t) u^{\prime 2}-\beta^{\prime \prime}(t) \frac{u^{2}}{2}\right] \\
& >\int_{0}^{2 \pi}\left[u^{\prime \prime 2}+q u^{\prime 2}-\left(a+\frac{r}{2}\right) u^{2}\right]
\end{aligned}
$$

which, by (iii), proves the required inequality.

5. EXTENSIONS

1) We can generalize Theorems 6 and 9 to the problem

$$
\begin{gathered}
\left(p u^{\prime \prime}\right)^{\prime \prime}=f\left(t, u, u^{\prime}, u^{\prime \prime}, u^{\prime \prime \prime}\right), \\
u(0)=u(2 \pi), u^{\prime}(0)=u^{\prime}(2 \pi), \\
\left(p u^{\prime \prime}\right)(0)=\left(p u^{\prime \prime}\right)(2 \pi),\left(p u^{\prime \prime}\right)^{\prime}(0)=\left(p \iota^{\prime \prime}\right)^{\prime}(2 \pi),
\end{gathered}
$$

where $p \in W^{2,1}(0,2 \pi)$ is such that $p(t)>0$ in $[0,2 \pi]$.

2) We can improve Theorem 11 by introducing in $f$ a linear dependence in $u^{\prime}$. We obtain for example the following result :

THEOREM 14. Let $f:[0,2 \pi] \times \mathbb{R}^{3} \rightarrow \mathbb{R}$ be a $L^{1}$-Carathéodory function. Assume that there exist $p, q, r \in \mathbb{R}, a>0, b, c \geq 0, \alpha, \delta \in L^{1}$ such that

(i) $|f(t, u, v, w)-,p u-r v-q w| \leq \alpha(t)|u|+b|v|+c|w|+\delta(t)$;

(ii) $\alpha(t)<a$;

(iii) there exist $\eta>c / 2, \xi>0$ such that for all $k \in \mathbb{N}$

$$
\begin{gathered}
k^{4}+q k^{2}-p \neq 0, \\
1 \geq \frac{c\left(\frac{k^{4}}{2 \eta}+\frac{\eta}{2}\right)+b\left(\frac{\xi}{2}+\frac{k^{2}}{2 \xi}\right)+a}{\left|k^{4}+q k^{2}-p\right|} .
\end{gathered}
$$

Then, the problem (4.3)-(4.4) has at least one solution.

3) We can consider, in a similar way, other boundary conditions as, for example,

$$
\begin{gathered}
u(0)=u(\pi)=0=u^{\prime}(0)=u^{\prime}(\pi) \\
u(0)=u(\pi)=0=u^{\prime \prime}(0)=u^{\prime \prime}(\pi) \\
u^{\prime}(0)=u^{\prime}(\pi)=0=u^{\prime \prime \prime}(0)=u^{\prime \prime \prime}(\pi) \\
u(0)=u^{\prime}(1)=0=u^{\prime \prime}(0)=u^{\prime \prime \prime}(1) \\
u(0)=u(\pi)=0=u^{\prime}(0)=u^{\prime \prime}(\pi)
\end{gathered}
$$

4) We can improve the abstract Theorem 2 in the following way. We do not need to assume that the operators $L, A, B$ and the operators of $\mathcal{F}(X, V)$ are symmetric but only that they satisfy for all $u, v \in X \cap H$

$$
\begin{aligned}
& \langle L u, P v\rangle=\langle L v, P u\rangle, \quad\langle A u, P v\rangle=\langle A v, P u\rangle \\
& \langle B u, P v\rangle=\langle B v, P u\rangle, \quad\langle S u, P v\rangle=\langle S v, P u\rangle
\end{aligned}
$$

for some continuous linear operator $P: H \rightarrow V^{\prime}$. Then we define $\overline{\mathcal{A}}$ by $\overline{\mathcal{A}}(u, v)=\langle A u, P v\rangle$; the other bilinear forms are defined in a similar way. The inequality $(2.2)$ will be replaced by

$$
\langle Q(\bar{u}+\tilde{u}), P \tilde{u}-P \bar{u}\rangle<\mathcal{D}(\bar{u}+\tilde{u}, \bar{u}+\tilde{u}) .
$$

When we study the problem (1.3)-(1.4) on $H^{2} \cap H_{0}^{1}$, we can obtain existence results by using this approach with $P u=-u^{\prime \prime}$. This is due to the fact that on $H^{2} \cap H_{0}^{1}$, the kernel of $P$ reduces to $\{0\}$. Unfortunately, this is not the case for the periodic problem. Still, the same idea can be used, but with the more complicated operator $P u=-u^{\prime \prime}+m u$ on $H_{2 \pi}^{2}$, with $m>0$. This leads to 
THEOREM 15. Let $f:|0,2 \pi| \times \mathbb{R}^{3} \rightarrow \mathbb{R}$ be measurable in $l \in|0,2 \pi|$ for all $X \in \mathbb{R}^{3}$ and contimuous in $X \in \mathbb{R}^{3}$ for a.e. $t \in[0,2 \pi \mid$. Assume that there exist $p, q \in \mathbb{R}, a>0, b \geq 0$, $\alpha, \beta, \gamma \in L^{1}\left((0,2 \pi), \mathbb{R}^{\dagger}\right)$ such that for all $\left(u, v^{\prime}, u^{\prime}\right) \in \mathbb{R}^{3}$ and a.e. $t \in[0,2 \pi]$,

(i) $|f(t, u, v, w)-p u-q u| \leq \alpha(t)|u|+|\beta(t)| u \mid+\gamma(t)$;

(ii) $\alpha(t)<a, \beta(t) \leq b$;

(iii) there exist $\eta, \xi \in \mathbb{R}, m>0$ such that for all $k \in \mathbb{N}$

$$
\begin{gathered}
k^{4}+q k^{2}-p \neq 0, \\
1 \geq \frac{a\left(\frac{k^{4}}{2 \xi}+\frac{\xi}{2}+m\right)+b\left(k^{4}+m \frac{k^{4}}{2 \eta}+\eta \frac{m}{2}\right)}{\left(k^{2}+m\right)\left|\left(k^{4}+q k^{2}-p\right)\right|} .
\end{gathered}
$$

Then the problem (4.3)-(4.4) has at least one solution.

Noreover, as we are working in $H_{2 \pi}^{3}$ we can mtroduce in $f$ a linear dependence in $u^{\prime \prime \prime}$.

5) Finally the ideas developped in this work can be used to study other boundary value problems. For example, the irlea of Theorem 6 can be applied to the problem

$$
\begin{gathered}
-u^{(6)}=f\left(t, u, u^{\prime}, u^{\prime \prime}, u^{\prime \prime \prime}, u^{(4)}\right), \\
u^{\prime}(0)=u^{\prime}(\pi)=u^{(3)}(0)=u^{(3)}(\pi)=u^{(5)}(0)=u^{(5)}(\pi)=0,
\end{gathered}
$$

with a function $f$ of the form

$$
f\left(t, u, u^{\prime}, u^{\prime \prime}, u^{\prime \prime \prime}, u^{(4)}\right)=\left(g_{2}(t, u) u^{\prime \prime}\right)^{\prime \prime}-\left(g_{1}(t, u) u^{\prime}\right)^{\prime}+g_{0}(t, u) u+q(t, u),
$$

whereas the idea of Theorem 12 can be applied to the third order boundary value problem

$$
\begin{gathered}
u^{\prime \prime \prime}=f\left(t, u, u^{\prime}, u^{\prime \prime}\right), \\
u(0)=u(2 \pi), u^{\prime}(0)=u^{\prime}(2 \pi), u^{\prime \prime}(0)=u^{\prime \prime}(2 \pi) .
\end{gathered}
$$

\section{REFERENCES}

1. A.R. AFTABIZADEH. Existence and Uniqueness Theorems for Fourth Order Boundary Value Problems, J. Math. Anal. Appl. 116 (1986), 415-426.

2. R.P. AGARWAL : On Fourth Order Boundary Value Problems Arising in Beam Analysis, Differential Integral Equations 2 (1989), 91-110.

3. H. BREZIS : Analyse fonctionnelle: Théorie et Applications, Masson (1983).

4. C. DE COSTER - C. FABRY - P. HABETS : An Abstract Approach to a Class of Nonlinear Boundary Value Problems, Differential Integral Equations 4 (1991), 1073-1087.

5. M.A. DEL PINO - R.F. MANASEVICH : Existence for a Fourth Order Boundary Value Problem under a two-parameter Nonresonance Condition, Proc. Amer. Math. $\underline{\text { Soc. } 112}$ (1991), 81-87.

6. C. FABRY - F. MUNYAMARERE : Existence of Solutions for Nonlinear Boundary Value Problems and Injectivity of Related Linear Differential Operators, Séminaire Math. 197, Louvain-la-Neuve, 1991.

7. C.P. GUPTA : Asymptotic Conditions for the Solvability of a Fourth Order Boundary Value Problem with Periodic Boundary Conditions, to appear.

8. C.P. GUPTA : A Nonlinear Boundary Value Problem Associated with the Static Equilibrium of an Elastic Beam Supported by Sliding Clamps, Internat. J. Math. Math. Sci. 12 (1989), 697-711.

9. C.P. GUPTA : A Fourth Order Nonlinear Boundary Value Problem and an Integral Type Sign Condition, Appl. Math. Comput. 35 (1990), 231-242.

10. C.P. GUPTA : Solvability of a Fourth Order Boundary Value Problem with Periodic Boundary Conditions, Internat. J. Math. Math. Sci. 11 (1988), 275-284. 
11 ('P. (iUP'TA : Integral Type Asymptotic Comditions for the Solvaloility of a Periodic Fourth Order Boundary Value Problem, Diff. Es(pu. and Applic. 1 (1988), 381-38x.

12. (:P. GUP'TA : Existence and Uniquene'ss Theorems for Some Fourth Order Fully Quasilinear Boundary Value Problems, to appear.

13. ('.P. GUP'TA - J. MAWHIN : Weighted B̈̈renvalue, Eigenfunctions and Boundary Value Problems for Fourth ()rder (Ordinary Differential Equations, to appear in Recent Trends in Ordinary Diff. Egul., World Scientific, Singapore.

14. J. MAWHIN : Points fixes, points criticules et problemes anx limites, Sém. Math. Sup. (1985).

15. J. MAWIIN - J.R. WARD : Nonresonance and Existence for Nomlinear Filliptic Boundary Value Problems, Nonlinear Anal. $\underline{5}$ (1981), 677-684.

16. J. MAWIIIN - J.R. WARD : Nonuniform Nonresonance Conditions at the two first Eigenvalues for Periodic Solutions of Forced Ijenard and Duffing Equations, Rocky Mountain $\underline{\mathrm{J}}$. Math. 12 (1982), 643-654.

17. G. METZEN : Semilinear Fourth Order Bounclary Value Problems, Bull. Austral. Math. Soc. $\underline{42}$ (1990), 101-114.

18. L. SANCIEZ : Boundary Value Problems for some Fourth Order Ordinary 1)ifferential Equations, Appl. Anal. 38 (1990), 161-177.

19. J.D. SCHUUR : Perturbation at Resonance for a Fourth Order Ordinary Differential Equation, J. Math. Anal. Appl. $\underline{65}$ (1978), 20-25.

20. R.A. USMANI : A Uniqueness Theorem for a Boundary Value Problem, Proc. Amer. Math. Soc. 77 (1979), 329-335.

21. M. WILLEM : Analyse Convexe et Optimisation, 3e édition, CIACO, Iouvain-la-Neuve (1989).

22. Y. YANG : Fourth Order Two-Point Boundary Value Problems, Proc. Amer. Math. Soc. 104 (1988), 175-180.

23. Y. YANG : Fourth Order Boundary Value Problems at Nonresonance, Bull. Austral. Math. Soc. $\underline{37}(1988), 337-343$. 


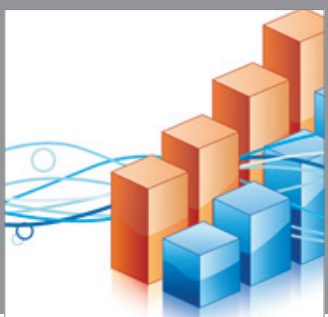

Advances in

Operations Research

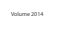

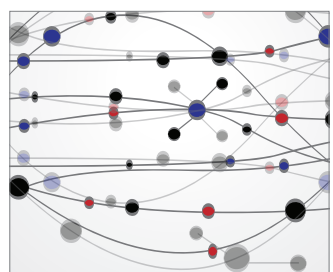

\section{The Scientific} World Journal
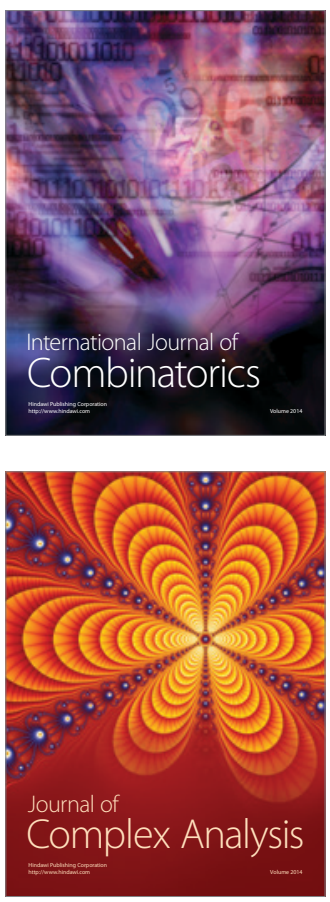

International Journal of

Mathematics and

Mathematical

Sciences
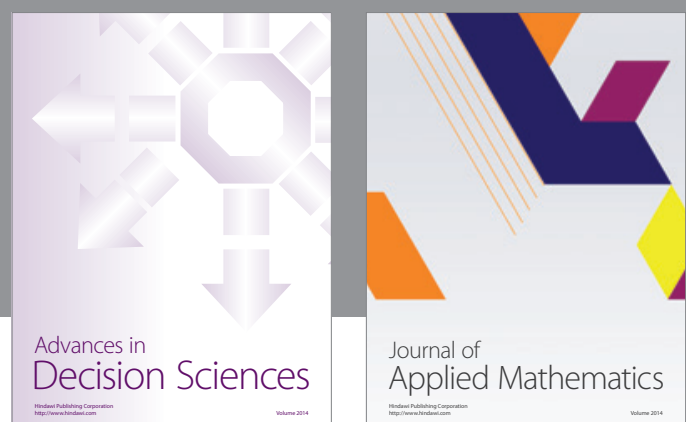

Journal of

Applied Mathematics
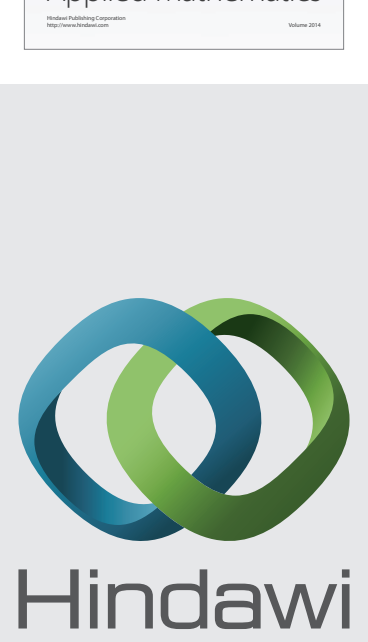

Submit your manuscripts at http://www.hindawi.com
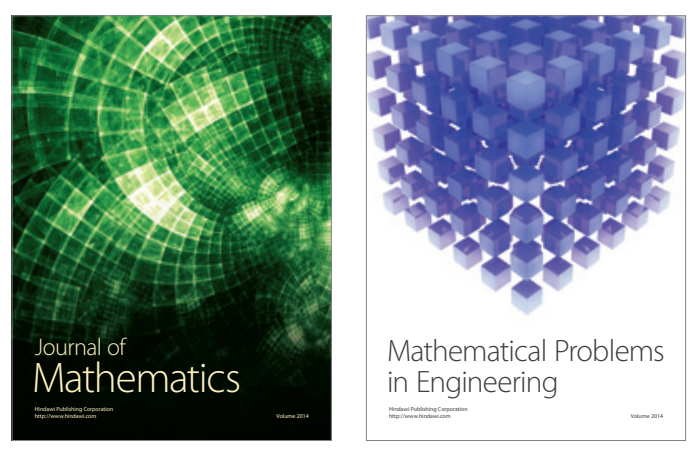

Mathematical Problems in Engineering
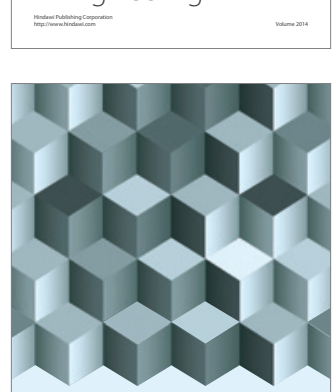

Journal of

Function Spaces
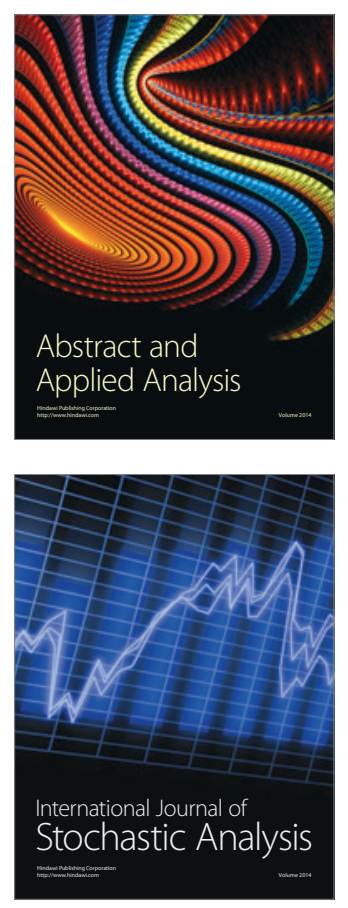

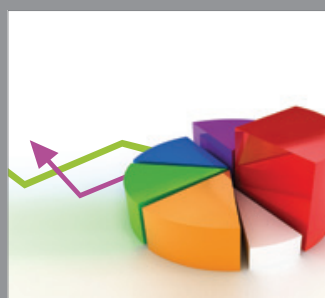

ournal of

Probability and Statistics

Promensencen
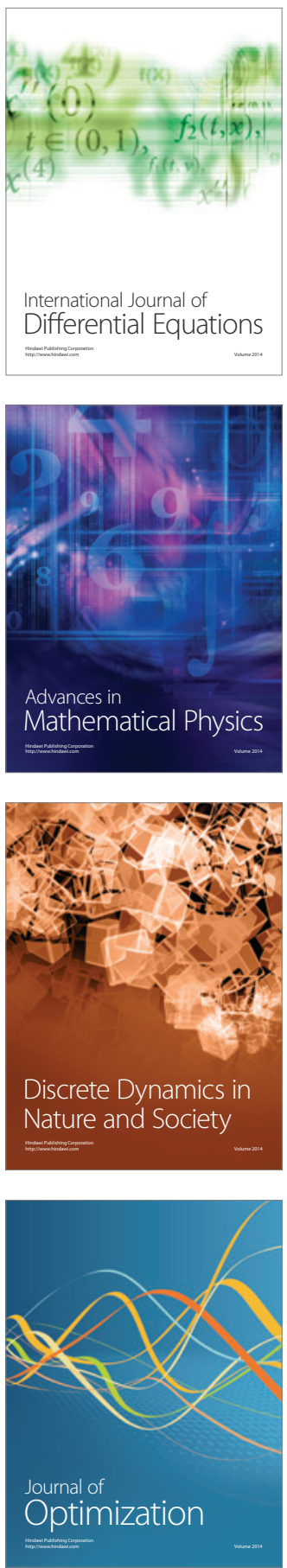